\title{
WEIGHTED GENERALIZED ESTIMATING EQUATIONS FOR RESPONSE-ADAPTIVE TREATMENT REGIMES IN TWO-STAGE LONGITUDINAL STUDIES
}

\author{
JESSE YENCHIH HSU* \\ Department of Biostatistics, Epidemiology and Informatics \\ University of Pennsylvania, Philadelphia, PA 19104, U.S.A. \\ Email: hsu9@upenn.edu \\ ABDus S. WAHED \\ Department of Biostatistics, University of Pittsburgh, Pittsburgh, PA 15261, U.S.A. \\ Email: wahed@pitt.edu
}

\section{SUMMARY}

\begin{abstract}
Two-stage longitudinal studies are common in the treatment of mental diseases, such as chronic forms of major depressive disorders. Outcomes in such studies often consist of repeated measurements of scores, such as the 24-item Hamilton Rating Scale for Depression, throughout the duration of therapy. Two issues that make the analysis of data from such two-stage studies different from standard longitudinal data are: (1) the randomization in the second stage for patients who fail to respond in the first stage; and (2) the drop-out of patients which sometimes occurs before the second stage. In this article, we show how the weighted generalized estimating equations can be used to draw inference for treatment regimes from two-stage studies. Specifically, we show how to construct weights and use them in the generalized estimating equations to derive consistent estimators of regime effects, and compare them. Large-sample properties of the proposed estimators are derived analytically, and examined through simulations. We demonstrate our methods by applying them to a depression dataset.
\end{abstract}

Keywords and phrases: Dynamic treatment regime; Generalized estimating equations; Inverse-probability-weighting; Two-stage design.

\section{Introduction}

In the past decade, a number of studies have shown the efficacy of pharmacotherapies and psychotherapies in treatment of chronic forms of major depressive disorders. However, nearly $50 \%$ of patients with chronic forms of major depressive disorders fail to respond to the first line pharmacotherapies or psychotherapies (Kocsis et al., 2009). Hence, for those who do not respond adequately to the first line therapy, combining pharmacotherapy and psychotherapy to replace monotherapy is

\footnotetext{
* Corresponding author

(c) Institute of Statistical Research and Training (ISRT), University of Dhaka, Dhaka 1000, Bangladesh.
} 
becoming more and more frequent in clinical practice. Combination treatment, in general, is relatively more expensive than monotherapy. A practical treatment strategy could be initially giving patients with chronic forms of major depressive disorders a common medication, which is usually cheaper, such as an antidepressant, and adding psychotherapy, which is more expensive, if patients have poor or partial response to the initial medication. Often, multiple stages of treatments may be necessary to achieve a response. A patient moves to the next stage of therapy in two circumstances: (1) if a patient achieves response, the patient could continue the same therapy or his/her therapy could be modified to maintain the response; and (2) if a patient fails to respond in the previous stage, the patient would be given some alternative therapies to achieve a response.

Between 2002 and 2006, the Research Evaluating the Value of Augmenting Medication with Psychotherapy (REVAMP) study (Trivedi et al., 2008) enrolled a total of 808 patients to determine the role of adjunctive psychotherapy in chronically depressed patients who had less than complete response to an initial medication. The study consisted of two 12-week stages. In stage one, patients were assigned one of four treatments of antidepressants by the REVAMP physicians based on a pharmacotherapy algorithm. These antidepressants were Sertaline (SERT), Escitalopram (EcCIT), Bupropion (BUP-SR), and Venlafaxine (VLF-XR). After up to 12 weeks of treatment in stage one, patients not meeting certain response criteria were randomized to three pharmacotherapies in stage two. These pharmacotherapies were Cognitive Behavioral Analysis System of Psychotherapy (CBASP), Brief Supportive Psychotherapy (BSP), and Medication alone (MED). For patients with full response to their antidepressant in stage one, the same antidepressant was given continuously in stage two. In both stages, a patient's 24-item Hamilton Rating Scale for Depression (Hamilton, 1960), was collected at each visit.

The purpose of the REVAMP study was to determine optimal adjunctive psychotherapies with which chronically depressed patients would benefit most. Since the adjunctive psychotherapies would be offered only if a chronically depressed patient fails to respond to the pharmacotherapy, possible choices of treatment regime would be to continue the same pharmacotherapy if a patient responds to the therapy and to choose a psychotherapy if the patient fails to respond to the pharmacotherapy. Because there would be different options of pharmacotherapies and psychotherapies, the practicing physicians would have to choose from many different treatment regimes. For example, in the REVAMP study, there were three possible treatment regimes: (1) treat with an antidepressant, continue the antidepressant if respond, otherwise add CBASP to the antidepressant; (2) treat with an antidepressant, continue the antidepressant if respond, otherwise add BSP to the antidepressant; and (3) treat with an antidepressant, continue the antidepressant if respond, otherwise add MED alone to the antidepressant. The aim of this study was to compare the efficacy of adding CBASP or BSP to continued treatment of antidepressants with MED alone. One naive way to analyze data from such designs would be to compare the psychotherapies conditional on the fact that the patients did not respond in stage one. However, this would be a conditional analysis and would not address the question of choosing an overall treatment regime to be best among all possible regimes.

The generalized estimating equations approach (Liang and Zeger, 1986; Zeger and Liang, 1986) is one of the well-known statistical methods to estimate the marginal treatment effect from longitudinal data. In a study with two stages of therapies, such as the REVAMP study, a patient can 
belong to several treatment regimes. For example, patients who had responded to the initial treatment would continue their treatment in stage two in the REVAMP study. These patients were treated consistently with all three regimes. Therefore, the standard generalized estimating equations may not be directly applicable. Additionally, patients who become eligible for the second stage treatments receive treatment by randomization and hence, the inverse-probability-weighted generalized estimating equations (Robins et al., 1995) can be used to account for the randomization. In recent years, many authors had studied the applications of the inverse-probability-weighted generalized estimating equations to estimate the effect of dynamic treatment regimes from sequential, multiple assignment, randomized trials (SMART). In Lu et al. (2016), the authors provided discussions on several SMART designs and modeling considerations for repeated measures in different degrees of complexity in SMART designs. In $\mathrm{Li}$ (2017), the authors estimated the randomization probabilities to improve the efficiency of the estimators for SMART designs and discussed various considerations to model the change of treatment effects over time. In this article, we develop analytical strategies for estimating treatment effects for dynamic treatment regimes to further consider patients who may drop out throughout the study. Drop-outs prior to observing the response status in stage one may be due to lack of efficacy to the first line therapy. Ignoring these patients in estimation might lead to biased estimates of effects of treatment regimes (Diggle et al., 2002). In this article, we use the inverse-probability-weighted generalized estimating equations to account for drop-outs. We derive consistent and asymptotically normal estimators of regime effects, and provide the Wald test for comparing different regimes.

\section{Data, Model, and Assumptions}

We consider a design that is more general than the REVAMP study. We assume that each patient $i, i=1, \ldots, n$, receives a first line treatment $A_{j}$ in the first stage, $j \in\{1, \ldots, J\}$. Patients who respond to $A_{j}$ are randomized to a maintenance treatment $B_{k}, k \in\{1, \ldots, K\}$, and patients who do not respond to $A_{j}$ are randomized to an alternative treatment $B_{l}^{\prime}, l \in\{1, \ldots, L\}$. The objective is to compare the effects of various treatment regimes arising from a combination of the initial treatment, intermediate response, and the second stage treatment. A treatment regime, $A_{j} B_{k} B_{l}^{\prime}$, is defined as: treat with $A_{j}$ followed by $B_{k}$ if respond, by $B_{l}^{\prime}$ otherwise. Patients are followed over time, and for patient $i$, a continuous outcome $Y_{i m}$ (e.g. the 24-item Hamilton Rating Scale for Depression) is measured at time $t_{i m}, m=1, \ldots, M_{i}$.

In the presence of randomization and drop-out, it is often useful to apply the idea of counterfactuals to the data analysis (Holland, 1986). For patient $i, i=1, \ldots, n$, define $R_{i}\left(A_{j}\right)$ to be the response status if the patient receives the first line treatment $A_{j}$; let $t_{i m_{1 i}}$ be the time when patient $i$ is declared a responder or non-responder to the first line treatment $A_{j}$, at which point randomization to the second set of treatment occurs, $m_{1 i} \in\left\{1, \ldots, M_{i}\right\}$. Whether observed or not, we define the following outcomes: $Y_{i}\left(A_{j}\right)$, a $m_{1 i} \times 1$ vector of repeated measures of outcome at time points in stage one if patient $i$ receives treatment $A_{j}$ in stage one; $Y_{i}\left(A_{j} B_{k}\right)$, a $\left(M_{i}-m_{1 i}\right) \times 1$ vector of repeated measures of outcome at each time point in stage two if patient $i$ receives treatment $A_{j}$ in stage one and $B_{k}$ in stage two after responding to $A_{j} ; Y_{i}\left(A_{j} B_{l}^{\prime}\right)$, a $\left(M_{i}-m_{1 i}\right) \times 1$ vector of repeated 
measures of outcome at each time point in stage two if patient $i$ receives treatment $A_{j}$ in stage one and $B_{l}^{\prime}$ in stage two after failing to respond to $A_{j}$. For simplicity, let us assume $J=K=L=2$. Thus, for one initial treatment $A_{1}$, patient $i$ could be associated with the following random variables:

$$
\left\{R_{i}\left(A_{1}\right),\left[1-R_{i}\left(A_{1}\right)\right], Y_{i}\left(A_{1}\right), Y_{i}\left(A_{1} B_{1}\right), Y_{i}\left(A_{1} B_{2}\right), Y_{i}\left(A_{1} B_{1}^{\prime}\right), Y_{i}\left(A_{1} B_{2}^{\prime}\right)\right\} .
$$

In terms of these counterfactual variables, let the $M_{i} \times 1$ vector outcome of patient $i$ under treatment regime $A_{1} B_{k} B_{l}^{\prime}$ for $k, l \in\{1,2\}$ be $Y_{i}\left(A_{1} B_{k} B_{l}^{\prime}\right)$. The m-th element of $Y_{i}\left(A_{1} B_{k} B_{l}^{\prime}\right)$ represents the outcome of patient $i$ at time $t_{i m}$ under regime $A_{1} B_{k} B_{l}^{\prime}$, and it can be expressed as:

$$
\begin{aligned}
Y_{i m}\left(A_{1} B_{k} B_{l}^{\prime}\right)=I\left(t_{i m}\right. & \left.\leq t_{i m_{1 i}}\right) Y_{i m}\left(A_{1}\right)+I\left(t_{i m}>t_{i m_{1 i}}\right) \\
\times & \left\{R_{i}\left(A_{1}\right) Y_{i\left(m-m_{1 i}\right)}\left(A_{1} B_{k}\right)+\left[1-R_{i}\left(A_{1}\right)\right] Y_{i\left(m-m_{1 i}\right)}\left(A_{1} B_{l}^{\prime}\right)\right\} .
\end{aligned}
$$

In a similar fashion, we can define the outcome, $Y_{i m}\left(A_{2} B_{k} B_{l}^{\prime}\right)$, for patient $i$ at time $t_{i m}$ given $A_{2} B_{k} B_{l}^{\prime}$. Therefore, without loss of generality, we will consider one treatment $A_{1}$ in stage one. Our interest is to estimate the effect of treatment regime $A_{1} B_{k} B_{l}^{\prime}$ on time, which is formulated as the coefficient $\beta_{1,1 \mathrm{kl}}$ of time in the marginal mean model:

$$
E\left[Y_{i m}\left(A_{1} B_{k} B_{l}^{\prime}\right) \mid t_{i m}, W_{i}\right]=\beta_{0,1 k l}+\beta_{1,1 k l} \times t_{i m}+\beta_{2,1 k l} W_{1 i}+\cdots+\beta_{p+1,1 k l} W_{p i},
$$

where $W_{i}=\left[W_{1 i}, \ldots, W_{p i}\right]^{T}$ is a $p \times 1$ vector of baseline covariates for patient $i$. In other words, we would like to estimate the parameters $\beta_{1 k l}=\left[\beta_{0,1 k l}, \ldots, \beta_{p+1,1 k l}\right]^{T}$. If $Y_{i m}\left(A_{1} B_{k} B_{l}^{\prime}\right)$ were observed for each patient in the sample, the generalized estimating equations could have been used to estimate these coefficients. However, in reality, we cannot observe the outcome $Y_{i m}\left(A_{1} B_{k} B_{l}^{\prime}\right)$ for all patients. For example, if a patient receives $A_{1}$, responds to $A_{1}$, and then receives $B_{k^{\prime}}$ where $k^{\prime} \neq$ $k$, we do not observe $Y_{i m}\left(A_{1} B_{k} B_{l}^{\prime}\right)$ for that patient. The complete observed data are characterized as the set of independent and identically distributed random list:

$$
\left\{R_{i}, R_{i} Z_{k i},\left(1-R_{i}\right) Z_{l i}^{\prime}, W_{i}, Y_{i}\right\} \quad(k, l \in\{1,2\}, i=1, \ldots, n),
$$

where $R_{i}=1$, if the patient is a responder, and $R_{i}=0$, if otherwise; $W_{i}=\left[W_{1 i}, \ldots, W_{p i}\right]^{T}$ is a $p \times 1$ vector of $p$ baseline covariates; $Z_{k i}$ and $Z_{l i}^{\prime}$ are the assignment indicators for treatment $B_{k}$ and $B_{l}^{\prime}$, respectively, for $k, l \in\{1,2\} ; Z_{1 i}=1(0)$ if patient $i$ is randomized to $B_{1}\left(B_{2}\right) ; Z_{1 i}^{\prime}=1(0)$ if patient $i$ is randomized to $B_{1}^{\prime}\left(B_{2}^{\prime}\right) ; Z_{2 i}$ and $Z_{2 i}^{\prime}$ satisfy $Z_{1 i}+Z_{2 i}=1$ and $Z_{1 i}^{\prime}+Z_{2 i}^{\prime}=1 ; Y_{i}$ is a $M_{i} \times 1$ vector of repeated observed outcome for patient $i$.

In order to draw inference on $Y_{i}\left(A_{1} B_{k} B_{l}^{\prime}\right)$ from observed data, the consistency assumption is required to connect observed data and counterfactuals (Rubin, 1974; Robins et al., 2000). The consistency assumption implies that the observed outcome is equal to the counterfactual outcome under treatment assignment consistent with the counterfactual. In other words, for $m=1, \ldots, M_{i}$,

$$
\begin{aligned}
Y_{i m}=I\left(t_{i m} \leq\right. & \left.t_{i m_{1 i}}\right) Y_{i m}\left(A_{1}\right)+I\left(t_{i m}>t_{i m_{1 i}}\right) \\
& \times\left\{R_{i} \sum_{k=1}^{2} Z_{k i} Y_{i\left(m-m_{1 i}\right)}\left(A_{1} B_{k}\right)+\left(1-R_{i}\right) \sum_{l=1}^{2} Z_{l i}^{\prime} Y_{i\left(m-m_{1 i}\right)}\left(A_{1} B_{l}^{\prime}\right)\right\} .
\end{aligned}
$$


Another frequently made assumption is the sequential randomization assumption which states that the probabilities of assigning treatment $B_{k}$ and $B_{l}^{\prime}$ do not depend on counterfactuals given the history of information collected prior to the randomization (Rubin, 1974; Robins, 1986):

$$
\left.\begin{array}{l}
P\left\{Z_{k i}=1 \mid R_{i}=1, W_{i}, Y_{i}\left(A_{1} B_{k} B_{l}^{\prime}\right)\right\}=P\left\{Z_{k i}=1 \mid R_{i}=1, W_{i}\right\} ; \\
P\left\{Z_{l i}^{\prime}=1 \mid R_{i}=0, W_{i}, Y_{i}\left(A_{1} B_{k} B_{l}^{\prime}\right)\right\}=P\left\{Z_{l i}^{\prime}=1 \mid R_{i}=0, W_{i}\right\} ; \\
\text { for } k, l \in\{1,2\} .
\end{array}\right\}
$$

In the REVAMP study, the second stage treatment assignment probabilities were constant. Therefore, we define $P\left\{Z_{k i}=1 \mid R_{i}=1, W_{i}\right\}=\eta_{k}$ and $P\left\{Z_{l i}^{\prime}=1 \mid R_{i}=0, W_{i}\right\}=\zeta_{l}$.

\section{Inference from Complete Data (No Drop-out)}

If everyone in our sample had followed the same treatment regime, $A_{1} B_{k} B_{l}^{\prime}$, where $k, l \in\{1,2\}$, we could have used the generalized estimating equations method (Liang and Zeger, 1986; Zeger and Liang, 1986) to estimate the regime effect, i.e. a $(p+2) \times 1$ vector of parameter $\beta_{1 k l}=$ $\left[\beta_{0,1 k l}, \ldots, \beta_{p+1,1 k l}\right]^{T}$. The generalized estimating equations in this case would be

$$
\sum_{i=1}^{n} X_{i}^{T} V_{i, 1 k l}^{-1}\left[Y_{i}\left(A_{1} B_{k} B_{l}^{\prime}\right)-X_{i} \beta_{1 k l}\right]=0,
$$

where $X_{i}^{T}=\left[x_{i 1}, \ldots, x_{i M_{i}}\right]$, and $x_{i m}^{T}=\left[1, t_{i m}, W_{i}^{T}\right]\left(m=1, \ldots, M_{i}\right) ; V_{i, 1 k l}=\phi A_{i, 1 k l}^{1 / 2} R(\alpha) A_{i, 1 k l}^{1 / 2}$; $R(\alpha)$ is an $M_{i} \times M_{i}$ working correlation matrix specified by $\alpha ; A_{i, 1 k l}$ is an $M_{i} \times M_{i}$ diagonal matrix with $v_{i m}\left(X_{i} ; \beta_{1 k l}\right)$ as the $\mathrm{m}$-th element, where $v_{i m}\left(X_{i}, \beta_{1 k l}\right)$ is the assumed working variance function of $Y_{i m}\left(A_{1} B_{k} B_{l}^{\prime}\right)$ and $\phi$ is the dispersion parameter.

However, not all patients followed the treatment regime $A_{1} B_{k} B_{l}^{\prime}$. Some patients in the study received treatments inconsistent with $A_{1} B_{k} B_{l}^{\prime}$, i.e. these patients randomized to receive other second stage treatment $B_{3-k}$ or $B_{3-l}^{\prime}, k, l \in\{1,2\}$. The data from these patients can be treated as missing data while estimating $\beta_{1 k l}$. Because of randomization, these patients with treatments inconsistent with $A_{1} B_{k} B_{l}^{\prime}$ are similar to those treated under treatment regime $A_{1} B_{k} B_{l}^{\prime}$. Thus, the inverse-probability-weighted method (Horvitz and Thompson, 1952; Rosenbaum and Rubin, 1983) can be used to account for the data that are missing by randomization. Patients randomized to treatment $B_{k}$ are weighted by $1 / \eta_{k}$ and patients randomized to treatment $B_{l}^{\prime}$ are weighted by $1 / \zeta_{l}$, where $\eta_{k}$ and $\zeta_{l}$ are the randomization probabilities in the second stage given the observed response status $R_{i}=1$ and $R_{i}=0$, respectively. This way, a patient randomized to treatment $B_{k}$ or $B_{l}^{\prime}$ counts for the patient as well as for $\left(1 / \eta_{k}-1\right)$ or $\left(1 / \zeta_{k}-1\right)$ similar patients who have missing data with respect to treatment regime $A_{1} B_{k} B_{l}^{\prime}$ (i.e. randomized to a second stage treatment other than $B_{k}$ or $B_{l}^{\prime}$.) This inverse probability of treatment weighting will be applied to create a pseudo-population from each patient who follows the policy $A_{1} B_{k} B_{l}^{\prime}$. The weight, $Q_{i, 1 k l}$, is thus defined to be a $M_{i} \times M_{i}$ diagonal matrix with each m-th diagonal element, $Q_{i m, 1 k l}$, defined as

$$
\left[\frac{R_{i} Z_{k i}}{\eta_{k}}+\frac{\left(1-R_{i}\right) Z_{l i}^{\prime}}{\zeta_{l}}\right]
$$


Note that $\eta_{k}$ and $\zeta_{l}$ are the known second stage randomization probabilities. For an alternative approach, the two randomization probabilities may be estimated from the data; see $\mathrm{Li}$ (2017) for more details. Given the treatment regime $A_{1} B_{k} B_{l}^{\prime}$, we use the inverse-probability-weighted generalized estimating equations method (Robins et al., 1995) with the weight $Q_{i, 1 k l}$ to estimate the regime effect $\beta_{1 k l}$. The weighted estimating equations is then

$$
\sum_{i=1}^{n} U_{i}\left(\beta_{1 k l}\right)=\sum_{i=1}^{n} X_{i}^{T} V_{i, 1 k l}^{-1} Q_{i, 1 k l}\left(Y_{i}-X_{i} \beta_{1 k l}\right)=0,
$$

where $Y_{i}, X_{i}$, and $V_{i, 1 k l}$ are defined as in (3.1). The solution of (3.2), $\hat{\beta}_{1 k l}$, can be obtained through the iterative algorithm (Liang and Zeger, 1986):

$$
\hat{\beta}_{1 k l}^{(r+1)}=\hat{\beta}_{1 k l}^{(r)}+\left(\sum_{i=1}^{n} X_{i}^{T} V_{i, 1 k l}^{-1} Q_{i, 1 k l} X_{i}\right)^{-1} \sum_{i=1}^{n} X_{i}^{T} V_{i, 1 k l}^{-1} Q_{i, 1 k l}\left[Y_{i}-X_{i} \hat{\beta}_{1 k l}^{(r)}\right]
$$

Lemma 3.1. Under sequential randomization assumption in (2.5),

$E\left[Q_{i, 1 k l} \mid X_{i}, Y_{i}\left(A_{1} B_{k} B_{l}^{\prime}\right)\right]=I_{M_{i}}$.

Proof. For $m=1, \ldots, M_{i}$, this follows since

$$
\begin{aligned}
& E\left[Q_{i m, 1 k l} \mid X_{i}, Y_{i}\left(A_{1} B_{k} B_{l}^{\prime}\right)\right] \\
& =E\left\{E\left[\frac{R_{i} Z_{k i}}{\eta_{k}}+\frac{\left(1-R_{i}\right) Z_{l i}^{\prime}}{\zeta_{l}} \mid R_{i}, X_{i}, Y_{i}\left(A_{1} B_{k} B_{l}^{\prime}\right)\right]\right\} \\
& =E\left\{\frac{R_{i}}{\eta_{k}} E\left[Z_{k i} \mid R_{i}, X_{i}, Y_{i}\left(A_{1} B_{k} B_{l}^{\prime}\right)\right]+\frac{1-R_{i}}{\zeta_{l}} E\left[Z_{l i}^{\prime} \mid R_{i}, X_{i}, Y_{i}\left(A_{1} B_{k} B_{l}^{\prime}\right)\right]\right\} \\
& =1 \quad \text { (by sequential randomization assumption). }
\end{aligned}
$$

Proposition 3.1. Under consistency assumption in (2.4) and sequential randomization assumption in (2.5), $\hat{\beta}_{1 k l}$ is a consistent estimator of $\beta_{1 k l}$.

Proof. First, by consistency assumption in (2.4), $Q_{i, 1 k l} Y_{i}=Q_{i, 1 k l} Y_{i}\left(A_{1} B_{k} B_{l}^{\prime}\right)$. Also, $\hat{\beta}_{1 k l}$ satisfies Equation (3.2), and therefore, to show that $\hat{\beta}_{1 k l}$ is consistent, it suffices to show that $E\left[U_{i}\left(\beta_{1 k l}\right)\right]=$ 
0. Now,

$$
\begin{aligned}
& E\left[U_{i}\left(\beta_{1 k l}\right)\right] \\
& =E\left[X_{i}^{T} V_{i, 1 k l}^{-1} Q_{i, 1 k l}\left(Y_{i}-X_{i} \beta_{1 k l}\right)\right] \\
& =E\left\{X_{i}^{T} V_{i, 1 k l}^{-1} Q_{i, 1 k l}\left[Y_{i}\left(A_{1} B_{k} B_{l}^{\prime}\right)-X_{i} \beta_{1 k l}\right]\right\} \quad \text { (by consistency assumption in (2.4)) } \\
& =E\left(E\left\{X_{i}^{T} V_{i, 1 k l}^{-1} Q_{i, 1 k l}\left[Y_{i}\left(A_{1} B_{k} B_{l}^{\prime}\right)-X_{i} \beta_{1 k l}\right] \mid X_{i}, Y_{i}\left(A_{1} B_{k} B_{l}^{\prime}\right)\right\}\right) \\
& =E\left\{X_{i}^{T} V_{i, 1 k l}^{-1}\left[Y_{i}\left(A_{1} B_{k} B_{l}^{\prime}\right)-X_{i} \beta_{1 k l}\right]\right\} \quad \text { (by Lemma 3.1) } \\
& =E\left(E\left\{\mathbf{X}_{i}^{T} \mathbf{V}_{i, 1 k l}^{-1}\left[Y_{i}\left(A_{1} B_{k} B_{l}^{\prime}\right)-X_{i} \beta_{1 k l}\right] \mid X_{i}\right\}\right) \\
& =E\left(X_{i}^{T} V_{i, 1 k l}^{-1}\left\{E\left[Y_{i}\left(A_{1} B_{k} B_{l}^{\prime}\right) \mid X_{i}\right]-X_{i} \beta_{1 k l}\right\}\right) \\
& =0 \quad(\text { by Equation (2.3)). }
\end{aligned}
$$

Proposition 3.2. Under consistency assumption in (2.4) and sequential randomization assumption in (2.5), $\hat{\beta}_{1 k l}$ is asymptotically normally distributed with mean $\beta_{1 k l}$ and variance $\Sigma / n$, where

$$
\left.\begin{array}{l}
\Sigma=C^{-1}\left(\phi, \alpha, \beta_{1 k l}\right) B\left(\phi, \alpha, \beta_{1 k l}\right) C^{-1}\left(\phi, \alpha, \beta_{1 k l}\right), \\
C\left(\phi, \alpha, \beta_{1 k l}\right)=E\left(X_{i}^{T} V_{i, 1 k l}^{-1} X_{i}\right), \quad \text { and } \\
B\left(\phi, \alpha, \beta_{1 k l}\right)=E\left[X_{i}^{T} V_{i, 1 k l}^{-1} Q_{i, 1 k l}\left(Y_{i}-X_{i} \beta_{1 k l}\right)\left(Y_{i}-X_{i} \beta_{1 k l}\right)^{T} Q_{i, 1 k l} V_{i, 1 k l}^{-1} X_{i}\right] .
\end{array}\right\}
$$

Proof. First, the estimator $\hat{\beta}_{1 k l}$ satisfies $\sum_{i=1}^{n} X_{i}^{T} V_{i, 1 k l}^{-1} Q_{i, 1 k l}\left(Y_{i}-X_{i} \hat{\beta}_{1 k l}\right)=0$. Expanding $\sum_{i=1}^{n} X_{i}^{T} V_{i, 1 k l}^{-1} Q_{i, 1 k l}\left(Y_{i}-X_{i} \hat{\beta}_{1 k l}\right)$ around $\beta_{1 k l}$ using Taylor's expansion and manipulating, we obtain

$$
\begin{aligned}
& n^{\frac{1}{2}}\left(\hat{\boldsymbol{\beta}}_{1 k l}-\boldsymbol{\beta}_{1 k l}\right) \\
& \quad=\left(\frac{1}{n} \sum_{i=1}^{n} X_{i}^{T} V_{i, 1 k l}^{-1} Q_{i, 1 k l} X_{i}\right)^{-1} n^{-\frac{1}{2}} \sum_{i=1}^{n} X_{i}^{T} V_{i, 1 k l}^{-1} Q_{i, 1 k l}\left(Y_{i}-X_{i} \beta_{1 k l}\right)+o_{p}(1),
\end{aligned}
$$

where $o_{p}(1)$ is a term that converges in probability to 0 as $n \rightarrow \infty$. Note also that

$$
\left(\frac{1}{n} \sum_{i=1}^{n} X_{i}^{T} V_{i, 1 k l}^{-1} Q_{i, 1 k l} X_{i}\right) \rightarrow E\left(X_{i}^{T} V_{i, 1 k l}^{-1} Q_{i, 1 k l} X_{i}\right)=E\left(X_{i}^{T} V_{i, 1 k l}^{-1} X_{i}\right)=C\left(\phi, \alpha, \beta_{1 k l}\right) .
$$

Therefore, from Equation (3.4), one can write

$$
n^{\frac{1}{2}}\left(\hat{\beta}_{1 k l}-\beta_{1 k l}\right)=n^{-\frac{1}{2}} \sum_{i=1}^{n} \psi_{i, 1 k l}+o_{p}(1)
$$


where $\psi_{i, 1 k l}=C^{-1}\left(\phi, \alpha, \beta_{1 k l}\right) X_{i}^{T} V_{i, 1 k l}^{-1} Q_{i, 1 k l}\left(Y_{i}-X_{i} \beta_{1 k l}\right)$ is known as the influence function of the estimator $\hat{\beta}_{1 k l}$, where $E\left(\psi_{i, 1 k l}\right)=0$ and $E\left(\psi_{i, 1 k l} \psi_{i, 1 k l}^{T}\right)$ is positive definite. From (3.5), we see that $\hat{\beta}_{1 k l}$ is an asymptotically linear estimator of $\beta_{1 k l}$. Applying the central limit theorem to (3.5), we deduce that $n^{1 / 2}\left(\hat{\beta}_{1 k l}-\beta_{1 k l}\right) \rightarrow \operatorname{MVN}(0, \Sigma)$, where $\Sigma=E\left(\psi_{i, 1 k l} \psi_{i, 1 k l}^{T}\right)$ is given in (3.3).

The asymptotic variance-covariance matrix of $\hat{\beta}_{1 k l}$ can be estimated by the empirical estimator

$$
\operatorname{vâr}\left(\hat{\beta}_{1 k l}\right)=\frac{1}{n} \hat{E}\left(\psi_{i, 1 k l} \psi_{i, 1 k l}^{T}\right)=\frac{1}{n^{2}} \sum_{i=1}^{n} \hat{\psi}_{i, 1 k l} \hat{\psi}_{i, 1 k l}^{T},
$$

or by the model-based estimator

$$
\operatorname{vâr}\left(\hat{\beta}_{1 k l}\right)=\frac{1}{n}\left[C_{n}^{-1}\left(\hat{\phi}, \hat{\alpha}, \hat{\beta}_{1 k l}\right) B_{n}\left(\hat{\phi}, \hat{\alpha}, \hat{\beta}_{1 k l}\right) C_{n}^{-1}\left(\hat{\phi}, \hat{\alpha}, \hat{\beta}_{1 k l}\right)\right] \text {, }
$$

where

$$
\begin{aligned}
& \hat{\psi}_{i, 1 k l}=C_{n}^{-1}\left(\hat{\phi}, \hat{\alpha}, \hat{\beta}_{1 k l}\right) X_{i}^{T} \hat{V}_{i, 1 k l}^{-1} Q_{i, 1 k l}\left(Y_{i}-X_{i} \hat{\beta}_{1 k l}\right), \\
& C_{n}\left(\hat{\phi}, \hat{\alpha}, \hat{\beta}_{1 k l}\right)=\frac{1}{n} \sum_{i=1}^{n} X_{i}^{T} \hat{V}_{i, 1 k l}^{-1} Q_{i, 1 k l} X_{i}, \\
& B_{n}\left(\hat{\phi}, \hat{\alpha}, \hat{\beta}_{1 k l}\right)=\frac{1}{n} \sum_{i=1}^{n} X_{i}^{T} \hat{V}_{i, 1 k l}^{-1} Q_{i, 1 k l}\left(Y_{i}-X_{i} \hat{\beta}_{1 k l}\right)\left(Y_{i}-X_{i} \hat{\beta}_{1 k l}\right)^{T} Q_{i, 1 k l} \hat{V}_{i, 1 k l}^{-1} X_{i}, \\
& \hat{V}_{i, 1 k l}=\hat{\phi} \hat{A}_{i, 1 k l}^{\frac{1}{2}} R(\hat{\alpha}) \hat{A}_{i, 1 k l}^{\frac{1}{2}}, \hat{A}_{i, 1 k l}=\operatorname{diag}\left\{v_{i m}\left(X_{i} ; \hat{\beta}_{1 k l}\right), m=1, \ldots, M_{i}\right\}, \\
& \hat{\phi}=\frac{\sum_{i=1}^{n} \sum_{m=1}^{M_{i}} \hat{e}_{i m}^{2}}{\sum_{i=1}^{n} \sum_{m=1}^{M_{i}} Q_{i m, 1 k l}-(p+2)}, \text { and } \hat{e}_{i m}=\sqrt{ } Q_{i m, 1 k l}\left(Y_{i m}-x_{i m}^{T} \hat{\beta}_{1 k l}\right) .
\end{aligned}
$$

The specific estimator of $\alpha$ depends on the working correlation structure, $R(\alpha)$ (Liang and Zeger, 1986). For example, if corr $\left[Y_{i m}\left(A_{1} B_{k} B_{l}^{\prime}\right), Y_{i(m+s)}\left(A_{1} B_{k} B_{l}^{\prime}\right)\right]=\alpha^{s}$ for $s=0, \ldots, M_{i}-m$, i.e. $R(\alpha)$ has an autoregressive correlation structure (AR $(1))$, then

$$
\hat{\alpha}=\frac{\sum_{i=1}^{n} \sum_{m<M_{i}-1} \hat{e}_{i m} \hat{e}_{i(m+1)}}{\left[N^{*}-(p+2)\right] \hat{\phi}},
$$

where $N^{*}=\sum_{i=1}^{n}\left(\sum_{m=1}^{M_{i}} Q_{i m, 1 k l}-1\right)$.

\section{Inference from Incomplete Data: Presence of Drop-out}

In Section 3, we used the inverse-probability-weighted generalized estimating equations method to deal with missing data due to randomization in stage two of the therapy. We assumed that there was no drop-out. However, in longitudinal studies, drop-out is a common phenomenon. The generalized estimating equations or the inverse-probability-weighted generalized estimating equations can provide valid estimates of parameters as long as drop-outs are missing completely at random (Robins 
et al., 1995). If drop-outs depend on observed data (e.g. responses from previous visits or baseline characteristics), they are not missing completely at random but missing at random (Little and Rubin, 2002). Therefore, we need to adjust our estimators from Section 3 to account for missing at random.

In the presence of drop-outs, the observed data from patient $i$ are

$$
\left\{W_{i}, \Delta_{i}, H_{i}\right\} \quad(k, l \in\{1,2\}, i=1, \ldots, n)
$$

where $\Delta_{i}=0$ if patient $i$ had dropped out of the study before completion, and $\Delta_{i}=1$ otherwise. When $\Delta_{i}=1, H_{i}=\left\{R_{i}, R_{i} Z_{k i},\left(1-R_{i}\right) Z_{l i}^{\prime}, Y_{i}\right\}$. When $\Delta_{i}=0, H_{i}$ contains post-baseline information observed prior to drop-out. Thus, when $\Delta_{i}=0$ and drop-out occurs at time $t_{i\left(d_{i}+1\right)}$, $Y_{i}$ is a $d_{i} \times 1$ vector, where $d_{i}<M_{i}$. To account for the data that are missing due to drop-out, one can extend the idea of inverse-probability-weighting described in section 3. Let us define the probability of a patient having complete data as $\pi_{i}=P\left\{\Delta_{i}=1 \mid W_{i}\right\}$, where $W_{i}$ is a vector of baseline covariates. Had this probability been known, each patient who had complete data would have been weighted by $1 / \pi_{i}$ to account for the patients who have incomplete data. We, therefore, modify the m-th diagonal element of the weight matrix $Q_{i, 1 k l}$ as follows:

$$
Q_{i m, 1 k l}\left(\pi_{i}\right)=\frac{\Delta_{i}}{\pi_{i}}\left[\frac{R_{i} Z_{k i}}{\eta_{k}}+\frac{\left(1-R_{i}\right) Z_{l i}^{\prime}}{\zeta_{l}}\right]
$$

and the weight matrix $Q_{i, 1 k l}\left(\pi_{i}\right)=\operatorname{diag}\left\{Q_{i 1,1 k l}\left(\pi_{i}\right), \ldots, Q_{i M_{i}, 1 k l}\left(\pi_{i}\right)\right\}$. However, $\pi_{i}$ is unknown and needs to be estimated. Let $\pi_{i}=G_{i}\left(\gamma ; W_{i}\right)$ be the postulated model for drop-out process defined by a set of parameters $\gamma$ and $\hat{\gamma}$ be a regular and asymptotically linear estimator of $\gamma$. For instance, $G_{i}\left(\gamma ; W_{i}\right)$ could be the logistic regression model, $G_{i}\left(\gamma ; W_{i}\right)=\left[1+\exp \left(-W_{i}^{T} \gamma\right)\right]^{-1}$, and $\hat{\gamma}$ could be the corresponding maximum likelihood estimator. The weight in (4.1) is then re-defined by replacing $\pi_{i}$ by $G_{i}\left(\hat{\gamma} ; W_{i}\right)$, i.e. the $\mathrm{m}$-th diagonal element of $Q_{i, 1 k l}(\hat{\gamma})$ is

$$
\frac{\Delta_{i}}{G_{i}\left(\hat{\gamma} ; W_{i}\right)}\left[\frac{R_{i} Z_{k i}}{\eta_{k}}+\frac{\left(1-R_{i}\right) Z_{l i}^{\prime}}{\zeta_{l}}\right]
$$

We have slightly abused the notation to denote this weight matrix by $Q_{i, 1 k l}(\hat{\gamma})$ instead of $Q_{i, 1 k l}\left(\hat{\pi}_{i}\right)$. Given a treatment regime $A_{1} B_{k} B_{l}^{\prime}$, the estimating equations for the inverse-probability-weighted generalized estimating equations is then

$$
\sum_{i=1}^{n} U_{i}^{*}\left(\beta_{1 k l}, \hat{\gamma}\right)=\sum_{i=1}^{n} X_{i}^{T} V_{i, 1 k l}^{-1} Q_{i, 1 k l}(\hat{\gamma})\left(Y_{i}-X_{i} \beta_{1 k l}\right)=0
$$

where $Y_{i}, X_{i}$, and $V_{i, 1 k l}$ are defined as in (3.1). Again, as before, the solution of (4.2), $\hat{\beta}_{1 k l}^{*}$, can be obtained through iterative algorithm.

Lemma 4.1. Under sequential randomization assumption in (2.5), and when $\pi_{i}=G_{i}\left(\gamma ; W_{i}\right)$ is known, $E\left[Q_{i, 1 k l}(\gamma) \mid X_{i}, Y_{i}\left(A_{1} B_{k} B_{l}^{\prime}\right)\right]=I_{M_{i}}$. 
Proof. Using iterated conditioning $\left(m=1, \ldots, M_{i}\right)$,

$$
\begin{aligned}
& E\left[Q_{i m, 1 k l}(\gamma) \mid X_{i}, Y_{i}\left(A_{1} B_{k} B_{l}^{\prime}\right)\right] \\
& =E\left\{E\left[\frac{\Delta_{i}}{G_{i}\left(\gamma ; W_{i}\right)} Q_{i m, 1 k l} \mid \Delta_{i}, R_{i}, X_{i}, Y_{i}\left(A_{1} B_{k} B_{l}^{\prime}\right)\right]\right\} \\
& =E\left\{\frac{\Delta_{i}}{G_{i}\left(\gamma ; W_{i}\right)} E\left[Q_{i m, 1 k l} \mid \Delta_{i}, R_{i}, X_{i}, Y_{i}\left(A_{1} B_{k} B_{l}^{\prime}\right)\right]\right\} \\
& =E\left[\frac{\Delta_{i}}{G_{i}\left(\gamma ; W_{i}\right)} \times 1\right] \\
& =\frac{1}{G_{i}\left(\gamma ; W_{i}\right)} \times \pi_{i} \\
& =1
\end{aligned}
$$

Lemma 4.2. Under consistency assumption in (2.4), sequential randomization assumption in (2.5), and when $\pi_{i}=G_{i}\left(\gamma ; W_{i}\right)$ is correctly specified, $E\left[U_{i}^{*}\left(\beta_{1 k l}, \gamma\right)\right]=0$.

Proof. Under consistency assumption in (2.4), $Q_{i, 1 k l}(\gamma) Y_{i}=Q_{i, 1 k l}(\gamma) Y_{i}\left(A_{1} B_{k} B_{l}^{\prime}\right)$. Thus,

$$
\begin{aligned}
& E\left[U_{i}^{*}\left(\beta_{1 k l}, \gamma\right)\right] \\
& =E\left[X_{i}^{T} V_{i, 1 k l}^{-1} Q_{i, 1 k l}(\gamma)\left(Y_{i}-X_{i} \beta_{1 k l}\right)\right] \\
& =E\left\{X_{i}^{T} V_{i, 1 k l}^{-1} Q_{i, 1 k l}(\gamma)\left[Y_{i}\left(A_{1} B_{k} B_{l}^{\prime}\right)-X_{i} \beta_{1 k l}\right]\right\} \quad \text { (by consistency assumption in (2.4)) } \\
& =E\left(E\left\{X_{i}^{T} V_{i, 1 k l}^{-1} Q_{i, 1 k l}(\gamma)\left[Y_{i}\left(A_{1} B_{k} B_{l}^{\prime}\right)-X_{i} \beta_{1 k l}\right] \mid X_{i}, Y_{i}\left(A_{1} B_{k} B_{l}^{\prime}\right)\right\}\right) \\
& =E\left\{X_{i}^{T} V_{i, 1 k l}^{-1}\left[Y_{i}\left(A_{1} B_{k} B_{l}^{\prime}\right)-X_{i} \beta_{1 k l}\right]\right\} \quad \text { (by Lemma 4.1) } \\
& =E\left(E\left\{X_{i}^{T} V_{i, 1 k l}^{-1}\left[Y_{i}\left(A_{1} B_{k} B_{l}^{\prime}\right)-X_{i} \beta_{1 k l}\right] \mid X_{i}\right\}\right) \\
& =E\left(X_{i}^{T} V_{i, 1 k l}^{-1}\left\{E\left[Y_{i}\left(A_{1} B_{k} B_{l}^{\prime}\right) \mid X_{i}\right]-X_{i} \beta_{1 k l}\right\}\right) \\
& =0 \quad(\text { by Equation }(2.3)) .
\end{aligned}
$$

Proposition 4.1. $\hat{\beta}_{1 k l}^{*}$ is a consistent estimator of $\beta_{1 k l}$, provided (1) $\hat{\gamma}$ is $\sqrt{ } n$-consistent, (2) $G_{i}\left(\gamma ; W_{i}\right)$ is specified correctly, and (3) the probability of having complete data does not depend on counterfactuals.

Proof. From (1), we can write $\sqrt{ } n(\hat{\gamma}-\gamma)=n^{-1 / 2} \sum_{i=1}^{n} \varphi_{i}(\gamma)+o_{p}(1)$, where $\varphi_{i}(\gamma)$ is the influence function of the estimator $\hat{\gamma}$ and $E\left[\varphi_{i}(\gamma)\right]=0$. Since $\hat{\beta}_{1 k l}^{*}$ satisfies Equation (4.2), the estimator $\left(\hat{\beta}_{1 k l}^{*}, \hat{\gamma}\right)^{T}$ is an M-estimator (Stefanski and Boos, 2002) defined by

$$
\sum_{i=1}^{n} \Psi_{i}\left(\beta_{1 k l}, \gamma\right)=\sum_{i=1}^{n}\left\{\begin{array}{c}
U_{i}^{*}\left(\beta_{1 k l}, \gamma\right) \\
\varphi_{i}(\gamma)
\end{array}\right\}=0
$$


The consistency of $\hat{\beta}_{1 k l}^{*}$ follows from the fact that $E\left[\Psi_{i}\left(\beta_{1 k l}, \gamma\right)\right]=0$ (by Lemma 4.2).

Proposition 4.2. Under assumptions similar to Proposition 4.1, $\hat{\beta}_{1 k l}^{*}$ is an asymptotically and normally distributed with mean $\beta_{1 k l}$ and variance $\Sigma^{*} / n$, where

$$
\left.\begin{array}{l}
\Sigma^{*}=C^{*^{-1}}\left(\phi, \alpha, \beta_{1 k l}, \gamma\right) B^{*}\left(\phi, \alpha, \beta_{1 k l}, \gamma\right) C^{*^{-1}}\left(\phi, \alpha, \beta_{1 k l}, \gamma\right) ; \\
C^{*}\left(\phi, \alpha, \beta_{1 k l}, \gamma\right)=E\left(X_{i}^{T} V_{i, 1 k l}^{-1} X_{i}\right) ; \\
B^{*}\left(\phi, \alpha, \beta_{1 k l}, \gamma\right) \\
=E\left[X_{i}^{T} V_{i, 1 k l}^{-1} Q_{i, 1 k l}(\gamma)\left(Y_{i}-X_{i} \beta_{1 k l}\right)\left(Y_{i}-X_{i} \beta_{1 k l}\right)^{T} Q_{i, 1 k l}(\gamma) V_{i, 1 k l}^{-1} X_{i}\right] \\
\quad-E\left[X_{i}^{T} V_{i, 1 k l}^{-1}\left(Y_{i}-X_{i} \beta_{1 k l}\right) \varphi_{i}^{T}(\gamma)\right] D^{*^{T}}\left(\phi, \alpha, \beta_{1 k l}, \gamma\right) \\
\quad-D^{*}\left(\phi, \alpha, \beta_{1 k l}, \gamma\right) E\left[\varphi_{i}(\gamma)\left(Y_{i}-X_{i} \beta_{1 k l}\right)^{T} V_{i, 1 k l}^{-1} X_{i}\right] \\
\quad+D^{*}\left(\phi, \alpha, \beta_{1 k l}, \gamma\right) E\left[\varphi_{i}(\gamma) \varphi_{i}^{T}(\gamma)\right] D^{*^{T}}\left(\phi, \alpha, \beta_{1 k l}, \gamma\right) ; \\
D^{*}\left(\phi, \alpha, \beta_{1 k l}, \gamma\right)=E\left[X_{i}^{T} V_{i, 1 k l}^{-1}\left(Y_{i}-X_{i} \beta_{1 k l}\right) G_{i}^{-1}\left(\gamma ; W_{i}\right) \frac{\partial}{\partial \gamma^{T}} G_{i}\left(\gamma ; W_{i}\right)\right]
\end{array}\right\}
$$

Proof. We start with the fact that the estimator $\hat{\beta}_{1 k l}^{*}$ satisfies $\sum_{i=1}^{n} U_{i}^{*}\left(\hat{\beta}_{1 k l}, \hat{\gamma}\right)=0$. First, expanding $\sum_{i=1}^{n} U_{i}^{*}\left(\hat{\beta}_{1 k l}, \hat{\gamma}\right)$ around $\beta_{1 k l}$ by Taylor's expansion and manipulating, we obtain

$$
n^{\frac{1}{2}}\left(\hat{\beta}_{1 k l}^{*}-\beta_{1 k l}\right)=\left[-\frac{1}{n} \sum_{i=1}^{n} \frac{\partial}{\partial \beta_{1 k l}^{T}} U_{i}^{*}\left(\beta_{1 k l}, \hat{\gamma}\right)\right]^{-1} n^{-\frac{1}{2}} \sum_{i=1}^{n} U_{i}^{*}\left(\beta_{1 k l}, \hat{\gamma}\right)+o_{p}(1)
$$

Then, applying Taylor's expansion on (4.4) around $\gamma$, it can be rewritten as

$$
\begin{aligned}
n^{\frac{1}{2}}\left(\hat{\beta}_{1 k l}^{*}-\beta_{1 k l}\right) & =\left[-\frac{1}{n} \sum_{i=1}^{n} \frac{\partial}{\partial \beta_{1 k l}^{T}} U_{i}^{*}\left(\beta_{1 k l}, \gamma\right)\right]^{-1} n^{-\frac{1}{2}} \sum_{i=1}^{n} U_{i}^{*}\left(\beta_{1 k l}, \gamma\right) \\
+ & \left\{\left[-\frac{1}{n} \sum_{i=1}^{n} \frac{\partial^{2}}{\partial \beta_{1 k l}^{T} \partial \gamma^{T}} U_{i}^{*}\left(\beta_{1 k l}, \gamma\right)\right]^{-1} \frac{1}{n} \sum_{i=1}^{n} U_{i}^{*}\left(\beta_{1 k l}, \gamma\right)\right. \\
+ & {\left.\left[-\frac{1}{n} \sum_{i=1}^{n} \frac{\partial}{\partial \beta_{1 k l}^{T}} U_{i}^{*}\left(\beta_{1 k l}, \gamma\right)\right]^{-1} \frac{1}{n} \sum_{i=1}^{n} \frac{\partial}{\partial \gamma^{T}} U_{i}^{*}\left(\beta_{1 k l}, \gamma\right)\right\} \times n^{\frac{1}{2}}(\hat{\gamma}-\gamma)+o_{p}(1) }
\end{aligned}
$$

Note the convergence of the following quantities in (4.5) as $n \rightarrow \infty$ :

$$
\begin{aligned}
& -\frac{1}{n} \sum_{i=1}^{n} \frac{\partial}{\partial \beta_{1 k l}^{T}} U_{i}^{*}\left(\beta_{1 k l}, \gamma\right) \rightarrow E\left(X_{i}^{T} V_{i, 1 k l}^{-1} X_{i}\right)=C^{*}\left(\phi, \alpha, \beta_{1 k l}, \gamma\right), \\
& \frac{1}{n} \sum_{i=1}^{n} U_{i}^{*}\left(\beta_{1 k l}, \gamma\right) \rightarrow 0 \quad \text { (by Lemma 4.2), and } \\
& \frac{1}{n} \sum_{i=1}^{n} \frac{\partial}{\partial \gamma^{T}} U_{i}^{*}\left(\beta_{1 k l}, \gamma\right) \rightarrow-E\left[X_{i}^{T} V_{i, 1 k l}^{-1}\left(Y_{i}-X_{i} \beta_{1 k l}\right) G_{i}^{-1}\left(\gamma ; W_{i}\right) \frac{\partial}{\partial \gamma^{T}} G_{i}\left(\gamma ; W_{i}\right)\right] .
\end{aligned}
$$


Using (4.6)-(4.8), we can write (4.5) as $n^{1 / 2}\left(\hat{\beta}_{1 k l}^{*}-\beta_{1 k l}\right)=n^{-1 / 2} \sum_{i=1}^{n} \psi_{i, 1 k l}^{*}+o_{p}(1)$, where $\psi_{i, 1 k l}^{*}$ is the influence function of the estimator $\hat{\beta}_{1 k l}^{*}$ and can be expressed as

$$
\begin{aligned}
\psi_{i, 1 k l}^{*}=C^{*^{-1}}\left(\phi, \alpha, \beta_{1 k l}, \gamma\right) & \\
& \times\left[X_{i}^{T} V_{i, 1 k l}^{-1} Q_{i, 1 k l}(\gamma)\left(Y_{i}-X_{i} \beta_{1 k l}\right)-D^{*}\left(\phi, \alpha, \beta_{1 k l}, \gamma\right) \varphi_{i}(\gamma)\right],
\end{aligned}
$$

where $D^{*}\left(\phi, \alpha, \beta_{1 k l}, \gamma\right)=E\left[X_{i}^{T} V_{i, 1 k l}^{-1}\left(Y_{i}-X_{i} \beta_{1 k l}\right) G_{i}^{-1}\left(\gamma ; W_{i}\right) \frac{\partial}{\partial \gamma^{T}} G_{i}\left(\gamma ; W_{i}\right)\right]$. By the central limit theorem, we can deduce that $n^{1 / 2}\left(\hat{\beta}_{1 k l}^{*}-\beta_{1 k l}\right) \rightarrow \operatorname{MVN}\left(0, \Sigma^{*}\right)$, where $\Sigma^{*}=E\left(\psi_{i, 1 k l}^{*} \psi_{i, 1 k l}^{* T}\right)$ and is given in (4.3).

The asymptotic variance-covariance matrix of $\hat{\beta}_{1 k l}^{*}$ can be estimated by the empirical estimator

$$
\operatorname{vâr}\left(\hat{\beta}_{1 k l}^{*}\right)=\frac{1}{n} \hat{E}\left(\psi_{i, 1 k l}^{*} \psi_{i, 1 k l}^{*^{T}}\right)=\frac{1}{n^{2}} \sum_{i=1}^{n} \hat{\psi}_{i, 1 k l}^{*} \hat{\psi}_{i, 1 k l}^{*^{T}},
$$

or by the model-based estimator

$$
\operatorname{vâr}\left(\hat{\beta}_{1 k l}^{*}\right)=\frac{1}{n} E\left[C_{n}^{*^{-1}}\left(\hat{\phi}, \hat{\alpha}, \hat{\beta}_{1 k l}^{*}, \hat{\gamma}\right) B_{n}^{*}\left(\hat{\phi}, \hat{\alpha}, \hat{\beta}_{1 k l}^{*}, \hat{\gamma}\right) C_{n}^{*^{-1}}\left(\hat{\phi}, \hat{\alpha}, \hat{\beta}_{1 k l}^{*}, \hat{\gamma}\right)\right],
$$

where

$$
\begin{aligned}
& \hat{\psi}_{i, 1 k l}^{*}=C_{n}^{*^{-1}}\left(\hat{\phi}, \hat{\alpha}, \hat{\beta}_{1 k l}^{*}, \hat{\gamma}\right)\left[X_{i}^{T} \hat{V}_{i, 1 k l}^{-1} Q_{i, 1 k l}(\hat{\gamma})\left(Y_{i}-X_{i} \hat{\beta}_{1 k l}^{*}\right)-D_{n}^{*}\left(\hat{\phi}, \hat{\alpha}, \hat{\beta}_{1 k l}^{*}, \hat{\gamma}\right) \varphi_{i}(\hat{\gamma})\right], \\
& C_{n}^{*}\left(\hat{\phi}, \hat{\alpha}, \hat{\beta}_{1 k l}^{*}, \hat{\gamma}\right)=\frac{1}{n} \sum_{i=1}^{n} X_{i}^{T} \hat{V}_{i, 1 k l}^{-1} Q_{i, 1 k l}(\hat{\gamma}) X_{i}, \\
& D_{n}^{*}\left(\hat{\phi}, \hat{\alpha}, \hat{\beta}_{1 k l}^{*}, \hat{\gamma}\right)=\frac{1}{n} \sum_{i=1}^{n}\left[X_{i}^{T} \hat{V}_{i, 1 k l}^{-1} Q_{i, 1 k l}(\hat{\gamma})\left(Y_{i}-X_{i} \hat{\beta}_{1 k l}^{*}\right) G_{i}^{-1}\left(\hat{\gamma} ; W_{i}\right) \dot{G}_{i}\left(\hat{\gamma} ; W_{i}\right)\right], \quad \text { and } \\
& B_{n}^{*}\left(\hat{\phi}, \hat{\alpha}, \hat{\beta}_{1 k l}^{*}, \hat{\gamma}\right)=\frac{1}{n} \sum_{i=1}^{n}\left[X_{i}^{T} \hat{V}_{i, 1 k l}^{-1} Q_{i, 1 k l}(\hat{\gamma})\left(Y_{i}-X_{i} \hat{\beta}_{1 k l}^{*}\right)\left(Y_{i}-X_{i} \hat{\beta}_{1 k l}^{*}\right)^{T} Q_{i, 1 k l}(\hat{\gamma}) \hat{V}_{i, 1 k l}^{-1} X_{i}\right] \\
& \quad-\left\{\frac{1}{n} \sum_{i=1}^{n}\left[X_{i}^{T} \hat{V}_{i, 1 k l}^{-1} Q_{i, 1 k l}(\hat{\gamma})\left(Y_{i}-X_{i} \hat{\beta}_{1 k l}^{*}\right) \varphi_{i}^{T}(\hat{\gamma})\right]\right\} \times D_{n}^{*}\left(\hat{\phi}, \hat{\alpha}, \hat{\beta}_{1 k l}^{* T}, \hat{\gamma}\right) \\
& \quad-D_{n}^{*}\left(\hat{\phi}, \hat{\alpha}, \hat{\beta}_{1 k l}^{*}, \hat{\gamma}\right) \times\left\{\frac{1}{n} \sum_{i=1}^{n}\left[\varphi_{i}(\hat{\gamma})\left(Y_{i}-X_{i} \hat{\beta}_{1 k l}^{*}\right)^{T} Q_{i, 1 k l}(\hat{\gamma}) \hat{V}_{i, 1 k l}^{-1} X_{i}\right]\right\} \\
& +D_{n}^{*}\left(\hat{\phi}, \hat{\alpha}, \hat{\beta}_{1 k l}^{*}, \hat{\gamma}\right) \times\left\{\frac{1}{n} \sum_{i=1}^{n}\left[\varphi_{i}(\hat{\gamma}) \varphi_{i}^{T}(\hat{\gamma})\right]\right\} \times D_{n}^{*^{T}}\left(\hat{\phi}, \hat{\alpha}, \hat{\beta}_{1 k l}^{*}, \hat{\gamma}\right) .
\end{aligned}
$$

The estimators of $\phi, \alpha, V_{i, 1 k l}$, and $A_{i, 1 k l}$ are obtained by replacing $\hat{\beta}_{1 k l}$ with $\hat{\beta}_{1 k l}^{*}$ and $Q_{i, 1 k l}$ with $Q_{i, 1 k l}(\hat{\gamma})$ in (3.6) and (3.7). 


\section{Comparison among Treatment Regimes}

To compare the effects of various regimes, contrasts of target regime effects can be constructed and tested via the Wald method. Since a patient can belong to more than one regime, the estimators of treatment effects among regimes will be correlated. In such situations, the covariance between estimators needs to be estimated. In the case of four treatment regimes, $A_{1} B_{k} B_{l}^{\prime}$ for $k, l \in\{1,2\}$, one can consider six pair-wise comparisons of the estimators of these four treatment regimes. Among these six pairs of the estimators, the covariance of $\left(\hat{\beta}_{111}^{*}, \hat{\beta}_{122}^{*}\right)$ will be zero, since $\hat{\beta}_{111}^{*}$ and $\hat{\beta}_{122}^{*}$ are estimated using data from two different subgroups of patients. For the same reason, the covariance of $\left(\hat{\beta}_{112}^{*}, \hat{\beta}_{121}^{*}\right)$ will also be zero. However, the estimators $\hat{\beta}_{111}^{*}$ and $\hat{\beta}_{112}^{*}$ are correlated, since both estimators used the same information from those patients who received $A_{1}$ and followed with $B_{1}$ after responding to $A_{1}$. Hence, the covariance of $\left(\hat{\beta}_{111}^{*}, \hat{\beta}_{112}^{*}\right)$ needs to be estimated. Similarly, we will have to estimate the covariances for the pairs $\left(\hat{\beta}_{111}^{*}, \hat{\beta}_{121}^{*}\right),\left(\hat{\beta}_{112}^{*}, \hat{\beta}_{122}^{*}\right)$, and $\left(\hat{\beta}_{121}^{*}, \hat{\beta}_{122}^{*}\right)$.

To estimate the covariance between two correlated estimators, we use the fact that the largesample covariance between two estimators can be obtained through the expectation of the product of their influence functions. We will demonstrate covariance computation for the pair of estimators $\hat{\beta}_{111}^{*}$ and $\hat{\beta}_{112}^{*}$. Similar computation follows for other correlated pairs. The covariance of $\hat{\beta}_{111}^{*}$ and $\hat{\beta}_{112}^{*}$ is $n^{-1} E\left(\psi_{i, 111}^{*} \psi_{i, 112}^{*^{T}}\right)$, where $\psi_{i, 111}^{*}$ and $\psi_{i, 112}^{*}$ are the influence functions of estimators $\hat{\beta}_{111}^{*}$ and $\hat{\beta}_{112}^{*}$, which are obtained from Equation (4.9). Therefore, one can estimate the covariance of $\hat{\beta}_{111}^{*}$ and $\hat{\beta}_{112}^{*}$ in a manner similar to the estimation of variance-covariance matrix through Equation (4.10) or (4.11).

To test the effects of treatment regimes $A_{1} B_{k} B_{l}^{\prime}$ for $k, l \in\{1,2\}, \beta=\left[\beta_{111}^{T}, \beta_{112}^{T}, \beta_{121}^{T}, \beta_{122}^{T}\right]^{T}$ via the Wald method, one can establish the null hypothesis of $A \beta=0$, where each $\beta_{1 k l}$ is a $(p+2)$ dimensional vector and $A$ is a matrix with $[4(p+2)]$ columns such that $\operatorname{rank}(A)<[4(p+2)]$. The test statistic will be

$$
T=(A \hat{\beta})^{T}\left(A \hat{\Sigma} A^{T}\right)^{-1}(A \hat{\beta}) \sim \chi_{\operatorname{rank}(A)}^{2},
$$

where $\hat{\beta}=\left[\hat{\beta}_{111}^{*^{T}}, \hat{\beta}_{112}^{*^{T}}, \hat{\beta}_{121}^{*^{T}}, \hat{\beta}_{122}^{*^{T}}\right]^{T}$ and $\hat{\Sigma}$ is the estimated covariance matrix of $\hat{\beta}$. Each element of $\hat{\Sigma}$ can be obtained by calculating the covariances of all pairs of estimators.

\section{Simulation Study}

To evaluate the performance of the estimators in small samples, we conducted several simulation studies. We simulated data from a population which has similar design to the REVAMP study. Each patient will have repeated measurements at each visit so that $t_{i}=[0,2,4,6,8,10,12]^{T}$ for all $i$. We considered one initial treatment $A_{1}$, two second stage treatments, $B_{1}$ and $B_{2}$, for responders, and two second stage treatments, $B_{1}^{\prime}$ and $B_{2}^{\prime}$, for non-responders. All patients received initial treatment $A_{1}$ at $t_{i 1}=0$. For each patient $i$, we generated counterfactual random variables listed in (2.1). At $t_{i 3}=4$ and $t_{i 4}=6$, patients were assessed to see if they had responded to the initial treatment $A_{1}$. Thus, the index of the time of response in this case is $m_{1 i}=3$ or 4 . The response status, $R_{i}$, was drawn from a $\operatorname{Bernoulli}(\theta)$ distribution. We assume that of these responders, $100 \times \theta_{1} \%$ responded at time $t_{i 3}$ and the rest at time $t_{i 4}$. Thus each responder was assigned randomly to respond 
at time $t_{i 3}$ with probability $\theta_{1}$. We considered two sets of parameters, $\left(\theta, \theta_{1}\right)=(0.5,0.25)$ and $(0.3,0.15)$ meaning $50 \%$ (30\%) of the patients would respond of which $25 \%(15 \%)$ would respond at time $t_{i 3}$ and the rest at time $t_{i 4}$. We also generated a baseline covariate, age, which follows a normal distribution with mean $\mu_{\text {age }}=45$ and standard deviation $\sigma_{\text {age }}=11$. For each patient $i$, counterfactual outcome vectors $Y_{i}\left(A_{1}\right), Y_{i}\left(A_{1} B_{1}\right), Y_{i}\left(A_{1} B_{2}\right), Y_{i}\left(A_{2} B_{1}\right)$, and $Y_{i}\left(A_{2} B_{2}^{\prime}\right)$, were generated as follows:

$$
\begin{aligned}
& Y_{i}\left(A_{1}\right) \sim \operatorname{MVN}_{m_{1 i}}\left(\left(\theta_{1, A_{1}}+\theta_{2, A_{1}} \times a g e_{i}\right) \times 1_{m_{1 i}}+\theta_{3, A_{1}} \times t_{0 i}, \Sigma_{0}\right), \\
& Y_{i}\left(A_{1} B_{1}\right) \sim \operatorname{MVN}_{7-m_{1 i}}\left(\left(\theta_{1, A_{1} B_{1}}+\theta_{2, A_{1} B_{1}} \times a g e_{i}\right) \times 1_{\left(7-m_{1 i}\right)}+\theta_{3, A_{1} B_{1}} \times t_{1 i}, \Sigma_{1}\right), \\
& Y_{i}\left(A_{1} B_{2}\right) \sim \operatorname{MVN}_{7-m_{1 i}}\left(\left(\theta_{1, A_{1} B_{2}}+\theta_{2, A_{1} B_{2}} \times a g e_{i}\right) \times 1_{\left(7-m_{1 i}\right)}+\theta_{3, A_{1} B_{2}} \times t_{1 i}, \Sigma_{1}\right), \\
& Y_{i}\left(A_{1} B_{1}^{\prime}\right) \sim \operatorname{MVN}_{7-m_{1 i}}\left(\left(\theta_{1, A_{1} B_{1}^{\prime}}+\theta_{2, A_{1} B_{1}^{\prime}} \times a g e_{i}\right) \times 1_{\left(7-m_{1 i}\right)}+\theta_{3, A_{1} B_{1}^{\prime}} \times t_{1 i}, \Sigma_{1}\right), \text { and } \\
& Y_{i}\left(A_{1} B_{2}^{\prime}\right) \sim \operatorname{MVN}_{7-m_{1 i}}\left(\left(\theta_{1, A_{1} B_{2}^{\prime}}+\theta_{2, A_{1} B_{2}^{\prime}} \times a g e_{i}\right) \times 1_{\left(7-m_{1 i}\right)}+\theta_{3, A_{1} B_{2}^{\prime}} \times t_{1 i}, \Sigma_{1}\right),
\end{aligned}
$$

where $m_{1 i}$ equals to either 3 or $4 ; t_{0 i}$ is a $m_{1 i} \times 1$ vector which indicates weeks of measurements in stage one and $t_{1 i}$ is a $\left(7-m_{1 i}\right) \times 1$ vector which indicates weeks of measurements in stage two; $\Sigma_{0}\left(\sigma_{0}, \rho_{0}\right)$ is a $m_{1 i} \times m_{1 i}$ covariance matrix defined by standard deviation $\sigma_{0}$ and autoregressive correlation $\rho_{0} ; \Sigma_{1}\left(\sigma_{1}, \rho_{1}\right)$ is a $\left(7-m_{1 i}\right) \times\left(7-m_{1 i}\right)$ covariance matrix defined by standard deviation $\sigma_{1}$ and autoregressive correlation $\rho_{1}$. The values of $\sigma_{0}, \sigma_{1}, \rho_{0}$, and $\rho_{1}$ were set to be $5,3,0.8$, and 0.8 , respectively; the parameters of correlations and standard deviations were estimated based on the choice of a correlation structure in the standard GEE procedure. For each patient $i$, the m-th element of the counterfactual outcome under a treatment regime $A_{1} B_{k} B_{l}^{\prime}$ for $k, l \in\{1,2\}$ and $m=1, \ldots, 7$ is generated using Equation (2.2). The estimation of parameters $\beta_{1 k l}=\left[\beta_{0,1 k l}, \beta_{1,1 k l}, \beta_{2,1 k l}\right]^{T}$ in Equation (2.3) of the model $E\left[Y_{i m}\left(A_{1} B_{k} B_{l}^{\prime}\right) \mid x_{i m}\right]=x_{i m}^{T} \beta_{1 k l}$ was the main focus in this article, where $x_{i m}=\left[1, t_{i m}, a e_{i}\right]^{T}$.

We considered the following parameter values for each counterfactual vector: $\theta_{1, A_{1}}=25, \theta_{2, A_{1}}=$ $0.5, \theta_{3, A_{1}}=-0.5, \theta_{1, A_{1} B_{1}}=27, \theta_{2, A_{1} B_{1}}=0.6, \theta_{3, A_{1} B_{1}}=-1.5, \theta_{1, A_{1} B_{2}}=38, \theta_{2, A_{1} B_{2}}=0.4, \theta_{3, A_{1} B_{2}}=-2$, $\theta_{1, A_{1} B_{1}^{\prime}}=36, \theta_{2, A_{1} B_{1}^{\prime}}=0.7, \theta_{3, A_{1} B_{1}^{\prime}}=-3, \theta_{1, A_{1} B_{2}^{\prime}}=68, \theta_{2, A_{1} B_{2}^{\prime}}=0.3$, and $\theta_{3, A_{1} B_{2}^{\prime}}=-5$. For this population, the true parameter values are $\beta_{111}^{T}=[23.09,-1.13,0.57], \beta_{112}^{T}=[27.94,-1.53,0.48]$, $\beta_{121}^{T}=[25.45,-1.30,0.52]$, and $\beta_{122}^{T}=[30.38,-1.69,0.43]$ for $50 \%$ response, and $\beta_{111}^{T}=$ $[22.88,-1.20,0.58], \beta_{112}^{T}=[29.65,-1.77,0.45], \beta_{121}^{T}=[24.28,-1.31,0.55]$ and $\beta_{122}^{T}=[31.10,-1.86,0.42]$ for $30 \%$ response.

We simulated 2000 Monte Carlo samples of sizes 250 and 400 observations from the populations described above with the following characteristics. For responders (i.e. $R_{i}=1$ ), the assignment indicator for treatment $B_{1}, Z_{1 i}$, was drawn from a Bernoulli distribution with probability $\eta_{1}$; the assignment indicator for treatment $B_{2}$ was defined as $Z_{2 i}=1-Z_{1 i}$. For non-responders, i.e. $R_{i}=0$, the assignment indicator for treatment $B_{1}^{\prime}, Z_{1 i}^{\prime}$ was drawn from a Bernoulli distribution with probability $\zeta_{1}$; the assignment indicator for treatment $B_{2}^{\prime}$ was defined as $Z_{2 i}^{\prime}=1-Z_{1 i}^{\prime}$. We defined the observed outcome for patient $i$ at $t_{i m}$ as in (2.4).

Additionally, a number of patients were allowed to drop out for the purpose of illustration based on the following logistic regression model: $\pi_{i}=G_{i}\left(\gamma ;\right.$ age,$\left.Y_{i 1}\right)=\operatorname{Pr}\left(\Delta_{i}=1 \mid\right.$ age, $\left.Y_{i 1}\right)=$ $\left[1+\exp \left(-\gamma_{0}-\gamma_{1} \times \text { age }-\gamma_{2} \times Y_{i 1}\right)\right]^{-1}$, where $\gamma=\left[\gamma_{0}, \gamma_{1}, \gamma_{2}\right]^{T} ; Y_{i 1}$ is the outcome at $t_{i 1}=0$ for patient $i$. Given this setting, the timing of individuals dropping out from the study would be 
uniformly across the study period. Choices of values of parameters $\gamma=[5.4,-0.02,-0.07]^{T}$ and $\gamma=[5.1,-0.03,-0.08]^{T}$ gave us approximate drop-out rates of $25 \%$ and $50 \%$, respectively. For sample sizes of 250 and 400 and response rates of $50 \%$ and $30 \%$, we considered the following dropout rates and analysis strategies: (1) no drop-out with weighting for randomization, $Q$; (2) $25 \%$ drop-out rate with weighting for randomization only. $Q$; (3) $25 \%$ drop-out rate with weighting for randomization and drop-out, $Q(\gamma)$; (4) $50 \%$ drop-out rate with weighting for randomization only, $Q$; (5) $50 \%$ drop-out rate with weighting for randomization and drop-out, $Q(\gamma)$.

Table 1 shows the simulation results of estimation for samples of size 250 . When the missing was due to randomization only, i.e. no drop-out, the inverse-probability-weighted generalized estimating equations estimators with $Q$ as weight matrix were approximately unbiased for all regimes regardless of the response rates. The maximum relative bias observed was $0.8 \%$. The standard deviations of the estimators were consistent with the Monte-Carlo standard deviations. The coverage probabilities for the $95 \%$ Wald confidence intervals for the parameters were between $92.4 \%$ and $95.7 \%$. For the $25 \%$ drop-out rate, the estimators were biased when the drop-out were ignored, i.e. used $Q$ as weight matrix in the inverse-probability-weighted generalized estimating equations. The relative biases ranged from 0.8 to $3.4 \%$. When the analysis accounted for drop-outs using $Q(\gamma)$ as weight matrix in the inverse-probability-weighted generalized estimating equations, the estimators were approximately unbiased. For example, the estimator weighted by $Q$ for regime $A_{1} B_{2} B_{2}^{\prime}$ with $30 \%$ response rate had relative bias $3.2 \%$, however, the estimator weighted by $Q(\gamma)$ for the same had near zero bias. The standard deviations of estimators weighted by $Q(\gamma)$ were consistent with corresponding Monte-Carlo standard deviations. The Wald confidence intervals achieved coverage close to their nominal confidence levels. When the drop-out rate was raised from $25 \%$ to $50 \%$, similar results were observed. The estimators weighted by $Q(\gamma)$ remained approximately unbiased for all regimes under both $50 \%$ and $30 \%$ response rates. Standard errors of estimators were slightly higher for higher drop-out rate.

For $n=400$ (data are shown in Table 2), the estimators with proper weighting were approximately unbiased regardless of response and drop-out rates. The standard errors of estimators were, as expected, smaller than those for $n=250$. The coverage probabilities for the $95 \%$ Wald confidence intervals ranged from $92.4 \%$ to $95.2 \%$.

\section{Analysis of the REVAMP Data}

A total of 808 patients with chronic forms of major depressive disorders were enrolled in the REVAMP study (Trivedi et al., 2008). Patients were evaluated in two 12-week stages for a maximum of 24 weeks. In stage one, patients were treated with one of four antidepressants: Sertaline (SERT), Escitalopram (EcCIT), Bupropion (BUP-SR), and Venlafaxine (VLF-XR). The choice of antidepressants was based on a pharmacotherapy algorithm (see Trivedi et al. (2008) for more details.) During week 8 through 12 in stage one, each patient was evaluated to assess the response. Patients with full response continued their antidepressant in stage two. Patients who did not meet the criteria of full response were randomized to three groups to additionally receive one of the following: Cognitive Behavioral Analysis System of Psychotherapy (CBASP), Brief Supportive Psychotherapy (BSP), or 
Table 1: Simulation results of estimation of $\beta_{1,1 k l}$ based on 2000 Monte Carlo samples of size 250. EST is Monte Carlo mean of estimates, SE is Monte Carlo mean of estimated standard errors, MCSE is standard error of Monte Carlo estimates, and CP is empirical coverage probability.

\begin{tabular}{|c|c|c|c|c|c|c|c|c|c|}
\hline $\begin{array}{c}\text { Drop-out } \\
\text { Rate }\end{array}$ & $\begin{array}{c}\text { Response } \\
\text { Rate }\end{array}$ & Regime & $\begin{array}{l}\text { True } \\
\text { Value }\end{array}$ & EST (SE) & MCSE & $\mathrm{CP} \%$ & EST (SE) & MCSE & $\mathrm{CP} \%$ \\
\hline & & & & \multicolumn{3}{|c|}{ Weighted by $Q$} & \multicolumn{3}{|c|}{ Weighted by $Q(\gamma)$} \\
\hline \multirow[t]{8}{*}{$0 \%$} & $50 \%$ & $A_{1} B_{1} B_{1}^{\prime}$ & -1.13 & $-1.13(0.050)$ & 0.050 & 95.7 & Not ap & plicable & \\
\hline & & $A_{1} B_{1} B_{2}^{\prime}$ & -1.53 & $-1.53(0.070)$ & 0.075 & 94.1 & Not ap & plicable & \\
\hline & & $A_{1} B_{2} B_{1}^{\prime}$ & -1.30 & $-1.30(0.047)$ & 0.049 & 92.8 & Not ap & plicable & \\
\hline & & $A_{1} B_{2} B_{2}^{\prime}$ & -1.69 & $-1.70(0.060)$ & 0.065 & 92.4 & Not ap & plicable & \\
\hline & $30 \%$ & $A_{1} B_{1} B_{1}^{\prime}$ & -1.20 & $-1.20(0.050)$ & 0.051 & 93.8 & Not ap & plicable & \\
\hline & & $A_{1} B_{1} B_{2}^{\prime}$ & -1.77 & $-1.76(0.067)$ & 0.069 & 94.3 & Not ap & plicable & \\
\hline & & $A_{1} B_{2} B_{1}^{\prime}$ & -1.31 & $-1.30(0.047)$ & 0.047 & 95.2 & Not ap & plicable & \\
\hline & & $A_{1} B_{2} B_{2}^{\prime}$ & -1.86 & $-1.86(0.058)$ & 0.060 & 94.6 & Not ap & plicable & \\
\hline \multirow[t]{8}{*}{$25 \%$} & $50 \%$ & $A_{1} B_{1} B_{1}^{\prime}$ & -1.13 & $-1.12(0.058)$ & 0.060 & 92.6 & $-1.13(0.056)$ & 0.054 & 96.2 \\
\hline & & $A_{1} B_{1} B_{2}^{\prime}$ & -1.53 & $-1.49(0.079)$ & 0.084 & 89.9 & $-1.53(0.082)$ & 0.088 & 95.9 \\
\hline & & $A_{1} B_{2} B_{1}^{\prime}$ & -1.30 & $-1.27(0.054)$ & 0.057 & 90.6 & $-1.31(0.053)$ & 0.056 & 92.1 \\
\hline & & $A_{1} B_{2} B_{2}^{\prime}$ & -1.69 & $-1.64(0.068)$ & 0.072 & 86.1 & $-1.71(0.068)$ & 0.070 & 94.4 \\
\hline & $30 \%$ & $A_{1} B_{1} B_{1}^{\prime}$ & -1.20 & $-1.19(0.058)$ & 0.058 & 93.9 & $-1.21(0.056)$ & 0.057 & 94.0 \\
\hline & & $A_{1} B_{1} B_{2}^{\prime}$ & -1.77 & $-1.71(0.075)$ & 0.079 & 87.4 & $-1.76(0.079)$ & 0.084 & 93.4 \\
\hline & & $A_{1} B_{2} B_{1}^{\prime}$ & -1.31 & $-1.28(0.055)$ & 0.056 & 91.7 & $-1.30(0.054)$ & 0.053 & 94.3 \\
\hline & & $A_{1} B_{2} B_{2}^{\prime}$ & -1.86 & $-1.80(0.066)$ & 0.069 & 82.2 & $-1.86(0.067)$ & 0.072 & 93.4 \\
\hline \multirow[t]{8}{*}{$50 \%$} & $50 \%$ & $A_{1} B_{1} B_{1}^{\prime}$ & -1.13 & $-1.10(0.071)$ & 0.070 & 91.8 & $-1.13(0.069)$ & 0.076 & 92.2 \\
\hline & & $A_{1} B_{1} B_{2}^{\prime}$ & -1.53 & $-1.44(0.093)$ & 0.093 & 86.2 & $-1.53(0.110)$ & 0.117 & 92.4 \\
\hline & & $A_{1} B_{2} B_{1}^{\prime}$ & -1.30 & $-1.24(0.067)$ & 0.072 & 83.4 & $-1.29(0.068)$ & 0.079 & 93.1 \\
\hline & & $A_{1} B_{2} B_{2}^{\prime}$ & -1.69 & $-1.58(0.081)$ & 0.087 & 66.8 & $-1.69(0.090)$ & 0.100 & 92.6 \\
\hline & $30 \%$ & $A_{1} B_{1} B_{1}^{\prime}$ & -1.20 & $-1.18(0.072)$ & 0.073 & 93.4 & $-1.20(0.070)$ & 0.075 & 93.5 \\
\hline & & $A_{1} B_{1} B_{2}^{\prime}$ & -1.77 & $-1.65(0.090)$ & 0.091 & 75.3 & $-1.76(0.105)$ & 0.110 & 92.6 \\
\hline & & $A_{1} B_{2} B_{1}^{\prime}$ & -1.31 & $-1.26(0.068)$ & 0.068 & 90.1 & $-1.30(0.068)$ & 0.070 & 94.0 \\
\hline & & $A_{1} B_{2} B_{2}^{\prime}$ & -1.86 & $-1.73(0.080)$ & 0.083 & 58.2 & $-1.86(0.088)$ & 0.095 & 92.8 \\
\hline
\end{tabular}


Table 2: Simulation results of estimation of $\beta_{1,1 k l}$ based on 2000 Monte Carlo samples of size 400. EST is Monte Carlo mean of estimates, SE is Monte Carlo mean of estimated standard errors, MCSE is standard error of Monte Carlo estimates, and $\mathrm{CP}$ is empirical coverage probability.

\begin{tabular}{|c|c|c|c|c|c|c|c|c|c|}
\hline $\begin{array}{c}\text { Drop-out } \\
\text { Rate }\end{array}$ & $\begin{array}{c}\text { Response } \\
\text { Rate }\end{array}$ & Regime & $\begin{array}{l}\text { True } \\
\text { Value }\end{array}$ & EST (SE) & MCSE & $\mathrm{CP} \%$ & EST (SE) & MCSE & $\mathrm{CP} \%$ \\
\hline & & & & \multicolumn{3}{|c|}{ Weighted by $Q$} & \multicolumn{3}{|c|}{ Weighted by $Q(\gamma)$} \\
\hline \multirow[t]{8}{*}{$0 \%$} & $50 \%$ & $A_{1} B_{1} B_{1}^{\prime}$ & -1.13 & $-1.13(0.042)$ & 0.042 & 95.5 & Not ap & plicable & \\
\hline & & $A_{1} B_{1} B_{2}^{\prime}$ & -1.53 & $-1.53(0.060)$ & 0.062 & 94.4 & Not ap & plicable & \\
\hline & & $A_{1} B_{2} B_{1}^{\prime}$ & -1.30 & $-1.30(0.040)$ & 0.041 & 93.9 & Not ap & plicable & \\
\hline & & $A_{1} B_{2} B_{2}^{\prime}$ & -1.69 & $-1.70(0.051)$ & 0.053 & 94.7 & Not ap & plicable & \\
\hline & $30 \%$ & $A_{1} B_{1} B_{1}^{\prime}$ & -1.20 & $-1.20(0.039)$ & 0.040 & 94.5 & Not ap & plicable & \\
\hline & & $A_{1} B_{1} B_{2}^{\prime}$ & -1.77 & $-1.76(0.053)$ & 0.055 & 94.1 & Not ap & plicable & \\
\hline & & $A_{1} B_{2} B_{1}^{\prime}$ & -1.31 & $-1.30(0.038)$ & 0.039 & 93.1 & Not ap & plicable & \\
\hline & & $A_{1} B_{2} B_{2}^{\prime}$ & -1.86 & $-1.86(0.046)$ & 0.048 & 94.6 & Not ap & plicable & \\
\hline \multirow[t]{8}{*}{$25 \%$} & $50 \%$ & $A_{1} B_{1} B_{1}^{\prime}$ & -1.13 & $-1.12(0.045)$ & 0.046 & 94.2 & $-1.13(0.044)$ & 0.045 & 92.4 \\
\hline & & $A_{1} B_{1} B_{2}^{\prime}$ & -1.53 & $-1.49(0.062)$ & 0.064 & 89.0 & $-1.53(0.065)$ & 0.065 & 95.2 \\
\hline & & $A_{1} B_{2} B_{1}^{\prime}$ & -1.30 & $-1.27(0.043)$ & 0.042 & 90.0 & $-1.30(0.042)$ & 0.044 & 94.5 \\
\hline & & $A_{1} B_{2} B_{2}^{\prime}$ & -1.69 & $-1.64(0.054)$ & 0.054 & 80.7 & $-1.69(0.055)$ & 0.057 & 94.6 \\
\hline & $30 \%$ & $A_{1} B_{1} B_{1}^{\prime}$ & -1.20 & $-1.19(0.046)$ & 0.046 & 94.3 & $-1.20(0.044)$ & 0.044 & 94.2 \\
\hline & & $A_{1} B_{1} B_{2}^{\prime}$ & -1.77 & $-1.71(0.060)$ & 0.062 & 83.0 & $-1.76(0.063)$ & 0.064 & 94.6 \\
\hline & & $A_{1} B_{2} B_{1}^{\prime}$ & -1.31 & $-1.29(0.043)$ & 0.044 & 91.4 & $-1.30(0.042)$ & 0.042 & 94.6 \\
\hline & & $A_{1} B_{2} B_{2}^{\prime}$ & -1.86 & $-1.80(0.052)$ & 0.056 & 76.6 & $-1.86(0.053)$ & 0.053 & 95.0 \\
\hline \multirow[t]{8}{*}{$50 \%$} & $50 \%$ & $A_{1} B_{1} B_{1}^{\prime}$ & -1.13 & $-1.11(0.056)$ & 0.057 & 92.5 & $-1.13(0.055)$ & 0.058 & 93.5 \\
\hline & & $A_{1} B_{1} B_{2}^{\prime}$ & -1.53 & $-1.44(0.074)$ & 0.074 & 77.6 & $-1.53(0.088)$ & 0.093 & 93.6 \\
\hline & & $A_{1} B_{2} B_{1}^{\prime}$ & -1.30 & $-1.24(0.052)$ & 0.056 & 79.1 & $-1.30(0.054)$ & 0.059 & 93.4 \\
\hline & & $A_{1} B_{2} B_{2}^{\prime}$ & -1.69 & $-1.57(0.065)$ & 0.066 & 48.8 & $-1.70(0.073)$ & 0.076 & 93.9 \\
\hline & $30 \%$ & $A_{1} B_{1} B_{1}^{\prime}$ & -1.20 & $-1.18(0.056)$ & 0.058 & 93.1 & $-1.20(0.056)$ & 0.056 & 94.8 \\
\hline & & $A_{1} B_{1} B_{2}^{\prime}$ & -1.77 & $-1.65(0.071)$ & 0.072 & 60.8 & $-1.76(0.084)$ & 0.089 & 94.5 \\
\hline & & $A_{1} B_{2} B_{1}^{\prime}$ & -1.31 & $-1.27(0.053)$ & 0.054 & 87.8 & $-1.30(0.054)$ & 0.056 & 93.3 \\
\hline & & $A_{1} B_{2} B_{2}^{\prime}$ & -1.86 & $-1.73(0.063)$ & 0.063 & 39.7 & $-1.86(0.070)$ & 0.073 & 93.8 \\
\hline
\end{tabular}




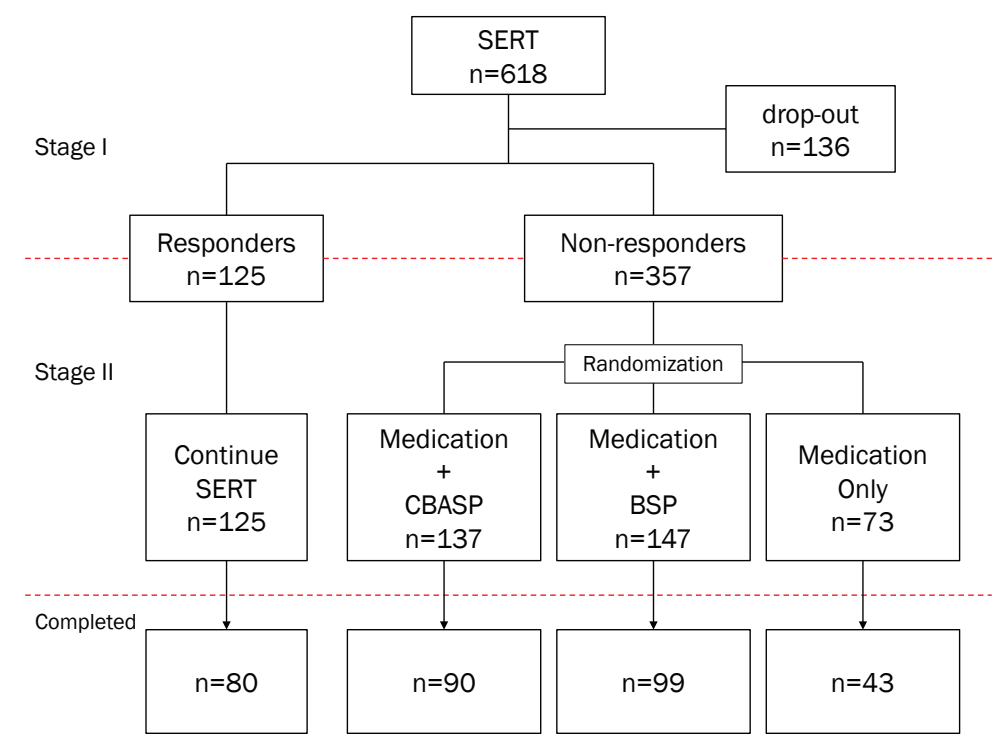

Figure 1: Patient flow in the REVAMP study

Medication alone (MED). The randomization was done with unequal probabilities of $0.4,0.4$, and 0.2 , respectively, to CBASP, BSP, and MED. For the purpose of illustration, we considered only patients treated with SERT in this article. Hence, we were interested in comparing three treatment regimes: (1) treat with SERT, continue SERT if respond, otherwise add CBASP to SERT; (2) treat with SERT, continue SERT if respond, otherwise add BSP to SERT; and (3) treat with SERT, continue SERT if respond, otherwise add MED to SERT. These three regimes are denoted by SSC, SSB, and SSM, respectively. The response to therapy was measured by the 24-item Hamilton Rating Scale for Depression $\left(\mathrm{HRSD}_{24}\right)$ scores at each visit. A reduction of $\mathrm{HRSD}_{24}$ score from baseline would indicate that the patient was recovering from depression. Figure 1 shows the design and patient flow of 618 patients in the REVAMP study who received SERT. The responses of 136 patients could not be assessed since they dropped out from the study in stage one. For 482 patients entering the second stage, about $65 \%$ of patients completed the study. To show a snapshot of the data, we have presented $\mathrm{HRSD}_{24}$ scores of eight selected patients in Figure 2. The left panel of Figure 2 represents $\mathrm{HRSD}_{24}$ scores for patients who had their response status confirmed at various time points and went on to complete the study. The right panel of Figure 2 represents $\mathrm{HRSD}_{24}$ scores for patients who dropped out from the study (their response statuses may or may not have been confirmed). Figure 2 illustrates how information collected in a two-stage longitudinal study can vary across patients due to response and drop-out. Because of the presence of drop-outs, we applied the inverse-probability-weighted generalized estimating equations method described in Section 4 to estimate the effects of three treatment regimes in the REVAMP study. The effect of the $j^{\text {th }}$ treatment regime was formulated as the 

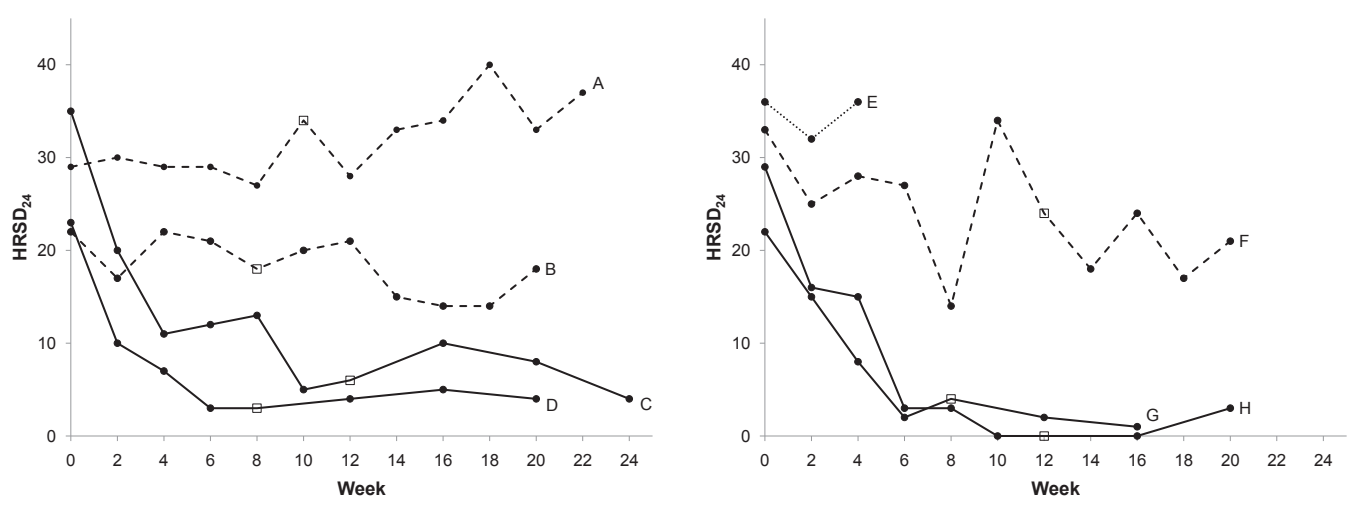

Figure 2: $\mathrm{HRSD}_{24}$ scores of eight selected patients in the REVAMP study. Patients A, B, C, and D had complete data and patients E, F, G, and H did not. Patients A and B are non-responders whose response status was confirmed at weeks 10 and 8, respectively. Patients $\mathrm{C}$ and $\mathrm{D}$ are responders whose response status was confirmed at weeks 12 and 8, respectively. Patient E dropped out at week 4 without response information. Patient $F$ dropped out at week 20 after failing to respond by week 12. Patients $\mathrm{G}$ and $\mathrm{H}$ dropped out at weeks 16 and 20 after responding to initial treatment at weeks 8 and 12, respectively. The solid lines represent responders, the dashed lines represent non-responders, and the dotted line represents patient who dropped out before the randomization and his/her response status could not be ascertained. The closed circles show when $\mathrm{HRSD}_{24} \mathrm{scores}$ were measured, the open squares show when the response status was confirmed.

coefficient $\beta_{t, j}$ in the marginal mean model:

$$
E\left[Y_{i m}(j) \mid t_{i m}, \text { gender }_{i}, \text { race }_{i}\right]=\beta_{0, j}+\beta_{t, j} \times t_{i m}+\beta_{\text {gender }, j} \times \text { gender }_{i}+\beta_{\text {race }, j} \times \text { race }_{i},
$$

where $j \in\{S S C, S S B, S S M\}$; gender $_{i}=1$ (0) if male (female); race $_{i}=1$ (0) if Caucasian (nonCaucasian). To account for incompleteness through the inverse-probability-weighted generalized estimating equations, the probability of having complete data for patient $i, \pi_{i}$, was estimated from the data. We postulated a logistic model for $\pi_{i}$ with covariates gender, race, and the baseline observed $\mathrm{HRSD}_{24}$ score. Specifically, $\hat{\pi}_{i}=G_{i}\left(\hat{\gamma} ; \operatorname{gender}_{i}, \operatorname{race}_{i}, Y_{i 1}\right)=\left[1+\exp \left(-\hat{\gamma}_{0}-\hat{\gamma}_{1} \times\right.\right.$ gender $_{i}-\hat{\gamma}_{2} \times$ race $\left.\left._{i}-\hat{\gamma}_{3} \times Y_{i 1}\right)\right]^{-1}$. Additionally, the exchangeable working correlation structure was used in the inverse-probability-weighted generalized estimating equations where the correlation and dispersion parameters were estimated by the standard GEE procedure.

The results of the REVAMP data analysis are shown in Table 3. The regime SSM had the highest reduction of 0.690 per week in $\mathrm{HRSD}_{24}$ scores from baseline, followed by the regimes SSB $(0.684 /$ week $)$ and SSC $(0.683 /$ week $)$. While the effect of each treatment regime was statistically significant $(\mathrm{p}<0.0001)$, the magnitude of the effect was similar across three regimes. A Wald Chisquare test $\left(H_{0}: \beta_{t, S S C}=\beta_{t, S S B}=\beta_{t, S S M}\right)$ with 2 degrees of freedom resulted in a p-value of 0.99 , implying that there was no evidence that the effects of these three treatment regimes were significantly different from each other. 
Table 3: The estimated effects of treatment regimes adjusting for gender and race in the REVAMP study

\begin{tabular}{lrrrrrr}
\hline $\begin{array}{l}\text { Treatment } \\
\text { Regime }\end{array}$ & $\begin{array}{r}\text { Estimated } \\
\text { Effect }\end{array}$ & $\begin{array}{r}\text { Standard } \\
\text { Error }\end{array}$ & $\begin{array}{c}\text { 95\% Confidence } \\
\text { Limits }\end{array}$ & $\begin{array}{c}\text { W-value } \\
\text { p-value df }=2\end{array}$ \\
\hline $\mathrm{SSC}^{1}$ & -0.683 & 0.027 & -0.736 & -0.630 & $<0.0001$ & \\
$\mathrm{SSB}^{2}$ & -0.684 & 0.029 & -0.741 & -0.626 & $<0.0001$ & 0.99 \\
$\mathrm{SSM}^{3}$ & -0.690 & 0.041 & -0.770 & -0.610 & $<0.0001$ & \\
\hline
\end{tabular}

${ }^{1}$ Treat with SERT, continue SERT if respond, otherwise add CBASP to SERT

${ }^{2}$ Treat with SERT, continue SERT if respond, otherwise add BSP to SERT

${ }^{3}$ Treat with SERT, continue SERT if respond, otherwise add MED to SERT

\section{Discussion}

In a two-stage longitudinal study, such as the one presented here, drop-out is a common phenomenon. If the drop-out occurs prior to the second randomization, a patient's response status will be unknown. Additionally, in the second stage of the study, patients randomized to one treatment can not receive other competing treatments, hence the fundamental problem of causal inference (Holland, 1986) applies. We have used the inverse-probability-weighted generalized estimating equations method (Robins et al., 1995) to take into account the missing data due to randomization and drop-out and have provided evidence of the bias incurred when appropriate weighting is not applied. The weights are formed by inversely weighting the probability of randomization to the treatment dictated by the regime and the probability of having complete data. The probability of having complete data is estimated through a logit model. We showed that under certain assumptions, the inverseprobability-weighted generalized estimating equations estimators are consistent and asymptotically normal. We also showed how to compare treatment regimes via the Wald test, which required computation of covariance between two estimated regime effects. We have demonstrated our methods using a dataset from a depression study.

Our methods account for missing data due to randomization and drop-out. However, they ignore the time to response and the time to drop-out in the second stage. It is possible to increase precision of the estimators by incorporating these two phenomenons into the estimation process. We are currently working on this generalization.

\section{References}

Diggle, P. J., Heagerty, P., Liang, K.-Y., and Zeger, S. L. (2002), Analysis of longitudinal data, New York: Oxford University Press.

Hamilton, M. (1960), “A rating scale for depression,” Journal of Neurology, Neurosugery, \& psychiatry, 23, 45-62. 
Holland, P. W. (1986), “Statistics and Causal Inference," Journal of the American Statistical Association, 81, 945-960.

Horvitz, D. G. and Thompson, D. J. (1952), "A generalization of sampling without replacement from a finite universe," Journal of the American Statistical Association, 47, 663-685.

Kocsis, J. H., Gelenberg, A. J., Rothbaum, B. O., Klein, D. N., Trivedi, M. H., Manber, R., Keller, M. B., Leon, A. C., Wisniewski, S. R., Arnow, B. A., Markowitz, J. C., Thase, M. E., and investigators, R. (2009), “Cognitive behavioral analysis system of psychotherapy and brief supportive psychotherapy for augmentation of antidepressant nonresponse in chronic depression: the REVAMP Trial," Archives of general psychiatry, 66, 1178-88.

Li, Z. (2017), "Comparison of adaptive treatment strategies based on longitudinal outcomes in sequential multiple assignment randomized trials," Statistics in Medicine, 36, 403-415.

Liang, K.-Y. and Zeger, S. L. (1986), "Longitudinal data analysis using generalized linear models," Biometrika, 73, 13-22.

Little, R. J. A. and Rubin, D. B. (2002), Statistical analysis with missing data, New York: Wiley.

Lu, X., Nahum-Shani, I., Kasari, C., Lynch, K. G., Oslin, D. W., Pelham, W. E., Fabianof, G., and Almirallb, D. (2016), "Comparing dynamic treatment regimes using repeated-measures outcomes: modeling considerations in SMART studies," Statistics in Medicine, 35, 1595-1615.

Robins, J. M. (1986), “A new approach to causal inference in mortality studies with a sustained exposure period - application to control of the healthy worker survivor effect," Mathematical Modelling, 7, 1393-1512.

Robins, J. M., Hernán, M. A., and Brumback, B. (2000), "Marginal Structural Models and Causal Inference in Epidemiology," Epidemiology, 11, 550-560.

Robins, J. M., Rotnitzky, A., and Zhao, L. P. (1995), "Analysis of semiparametric regression models for repeated outcomes in the presence of missing data," Journal of the American Statistical Association, 90, 106-121.

Rosenbaum, P. R. and Rubin, D. B. (1983), "The central role of the propensity score in observational studies for causal effects," Biometrika, 70, 41-55.

Rubin, D. B. (1974), "Estimating causal effects of treatments in randomized and nonrandomized studies," Journal of Educational Psychology, 66, 688-701.

Stefanski, L. A. and Boos, D. D. (2002), "The Calculus of M-Estimation," The American Statistician, 56, 29-38.

Trivedi, M. H., Kocsis, J. H., Thase, M. E., Horris, D. W., Wisniewski, S. R., Leon, A. C., Gelenberg, A. J., Klein, D. N., Niederehe, G., Schatzberg, A. F., Ninan, P. T., and Keller, M. B. (2008), "REVAMP - Research Evaluating the Value of Augmenting Medication with Psychotherapy: rationale and design," Psychopharmacology Bulletin, 41, 5-33. 
Zeger, S. L. and Liang, K.-Y. (1986), "Longitudinal Data Analysis for Discrete and Continuous Outcomes," Biometrics, 42, 121-130.

Received: May 27, 2017

Accepted: July 9, 2017 